ANNALES

POLONICI MATHEMATICI

$83.1(2004)$

\title{
Hukuhara's differentiable iteration semigroups of linear set-valued functions
}

\author{
by AndRzEJ SMAJdor (Kraków)
}

\begin{abstract}
Let $K$ be a closed convex cone with nonempty interior in a real Banach space and let $c c(K)$ denote the family of all nonempty convex compact subsets of $K$. A family $\left\{F^{t}: t \geq 0\right\}$ of continuous linear set-valued functions $F^{t}: K \rightarrow c c(K)$ is a differentiable iteration semigroup with $F^{0}(x)=\{x\}$ for $x \in K$ if and only if the set-valued function $\Phi(t, x)=F^{t}(x)$ is a solution of the problem

$$
D_{t} \Phi(t, x)=\Phi(t, G(x)):=\bigcup\{\Phi(t, y): y \in G(x)\}, \quad \Phi(0, x)=\{x\},
$$

for $x \in K$ and $t \geq 0$, where $D_{t} \Phi(t, x)$ denotes the Hukuhara derivative of $\Phi(t, x)$ with respect to $t$ and $G(x):=\lim _{s \rightarrow 0+}\left(F^{s}(x)-x\right) / s$ for $x \in K$.
\end{abstract}

1. Let $X$ be a vector space. Throughout this paper all vector spaces are supposed to be real. We write

$$
A+B=\{a+b: a \in A, b \in B\}, \quad \lambda A:=\{\lambda a: a \in A\}
$$

for $A, B \subset X$ and $\lambda \in \mathbb{R}$. A subset $K \subset X$ is called a cone if $t K \subset K$ for all positive $t$.

Let $X$ and $Y$ be two vector spaces and let $K \subset X$ be a convex cone. A set-valued function $F: K \rightarrow n(Y)$, where $n(Y)$ denotes the family of all nonempty subsets of $Y$, is called linear if

$$
F(x)+F(y)=F(x+y), \quad F(\lambda x)=\lambda F(x)
$$

for all $x, y \in K$ and $\lambda>0$.

Let $K$ be a convex cone in a normed vector space and let $b(K), c(K)$, and $c c(K)$ denote the sets of all bounded, compact, and convex compact members of $n(K)$, respectively. The difference $A-B$ of $A, B \in c c(K)$ is a set $C \in c c(K)$ such that $A=B+C$. If the difference exists, then it is unique. This is a consequence of a theorem of Rådström (see [7]).

Let $H:[0, \infty) \rightarrow c c(K)$ be a set-valued function such that the differences $H(t)-H(s)$ exist for $t, s \in[0, \infty)$ such that $t>s$. The Hukuhara derivative

2000 Mathematics Subject Classification: 39B12, 26E25.

Key words and phrases: linear set-valued functions, iterations, Hukuhara's derivative. 
of $H$ at $t$ is defined by the formula (see [3])

$$
D H(t)=\lim _{s \rightarrow t+} \frac{H(s)-H(t)}{s-t}=\lim _{s \rightarrow t-} \frac{H(t)-H(s)}{t-s},
$$

whenever both limits exist with respect to the Hausdorff metric $d$ in $c c(K)$ derived from the norm in $X$. Moreover,

$$
D H(0)=\lim _{s \rightarrow 0+} \frac{H(s)-H(0)}{s} .
$$

Now, we will prove the following

Lemma 1. Let $X$ be a Banach space and $H:[0, \infty) \rightarrow c c(X)$ be a setvalued function. If $H$ is differentiable at $t \in[0, \infty)$, then $H$ is continuous at this point.

Proof. For $s>t \geq 0$ we have

$$
\begin{aligned}
d(H(s), H(t)) & =d(H(s)-H(t),\{0\})=(s-t) d\left(\frac{H(s)-H(t)}{s-t},\{0\}\right) \\
& \leq(s-t)\left[d\left(\frac{H(s)-H(t)}{s-t}, D H(t)\right)+d(D H(t),\{0\})\right] .
\end{aligned}
$$

This implies that

$$
\lim _{s \rightarrow t+} H(s)=H(t) .
$$

Similarly, for $t>0$ and $0<s<t$ we have

$$
\begin{aligned}
d(H(s), H(t)) & =d(\{0\}, H(t)-H(s))=(t-s) d\left(\{0\}, \frac{H(t)-H(s)}{t-s}\right) \\
& \leq(t-s)\left[d\left(\frac{H(t)-H(s)}{t-s}, D H(t)\right)+d(D H(t),\{0\})\right],
\end{aligned}
$$

whence

$$
\lim _{s \rightarrow t-} H(s)=H(t)
$$

Thus $H$ is continuous at $t$.

2. Let $K$ be a nonempty set. A family $\left\{F^{t}: t \geq 0\right\}$ of set-valued functions $F^{t}: K \rightarrow n(K)$ is said to be an iteration semigroup if

$$
F^{s+t}(x)=F^{t}\left[F^{s}(x)\right]:=\bigcup\left\{F^{t}(y): y \in F^{s}(x)\right\}
$$

for all $x \in K$ and $t, s \geq 0$.

EXAmple 1 . Let $G: \mathbb{R}^{n} \rightarrow c c\left(\mathbb{R}^{n}\right)$ be a set-valued function. The attainable set $R(t, \xi)$ of the differential inclusion

$(*) \quad x^{\prime}(s) \in G(x(s)) \quad$ a.e. in $[0, t], \quad x(\cdot) \in A C[0, t], \quad x(0)=\xi$,

at time $t$ from $\xi \in \mathbb{R}^{n}$ is defined by the formula

$$
R(t, \xi)=\{x(t): x(\cdot) \text { satisfies }(*) \text { and } x(0)=\xi\} .
$$


It is known that if the sets $R(t, \xi)$ are nonempty, then the set-valued functions $\xi \mapsto R(t, \xi)$ form an iteration semigroup (see e.g. [2]). Moreover, if $G$ is locally Lipschitz on $\mathbb{R}^{n}$ and the sets $R([0, t], \xi)$ are compact, then $G$ is the infinitesimal generator of this semigroup (see [13]).

Let $K$ be a convex cone in a normed space. An iteration semigroup $\left\{F^{t}\right.$ : $t \geq 0\}$ of set-valued functions $F^{t}: K \rightarrow c c(K)$ is said to be differentiable if all set-valued functions $t \mapsto F^{t}(x)(x \in K)$ have Hukuhara's derivative on $[0, \infty)$.

Example 2. Let $K$ be closed convex cone with nonempty interior in a Banach space. Every concave iteration semigroup $\left\{F^{t}: t \geq 0\right\}$ of continuous linear set-valued functions $F^{t}: K \rightarrow c c(K)$ with $F^{0}(x)=\{x\}$ for $x \in K$ is differentiable (see [10]).

ExAmple 3 . The family $\left\{F^{t}: t \geq 0\right\}$, where $F^{t}(x)=\left[e^{t}, e^{2 t}\right] x$ for $t \in[0, \infty)$ and $x \in \mathbb{R}$, is a differentiable iteration semigroup of continuous linear set-valued functions. This semigroup is not concave.

Let $X$ and $Y$ be two vector spaces and let $K$ be a convex cone in $X$. A set-valued function $F: K \rightarrow n(Y)$ is called superadditive if

$$
F(x)+F(y) \subset F(x+y)
$$

for all $x, y \in K$. A set-valued function $F: K \rightarrow n(K)$ is said to be $\mathbb{Q}_{+}$homogeneous if

$$
F(\lambda x)=\lambda F(x)
$$

for all $x \in K$ and all positive rational numbers $\lambda$.

We will use the following six lemmas.

Lemma 2 (see Lemma 3 in [10]). Let $X$ and $Y$ be two topological vector spaces and let $K$ be a closed convex cone in $X$. Assume that $F: K \rightarrow$ $c c(K)$ is a continuous additive set-valued function and $A, B \in c c(K)$. If the difference $A-B$ exists, then $F(A)-F(B)$ exists and $F(A)-F(B)=$ $F(A-B)$.

Lemma 3 (Theorem 3 in [12], see also Lemma 4 in [9]). Let $X$ and $Y$ be two normed vector spaces and let $K$ be a convex cone in $X$. Suppose that $\left\{F_{i}: i \in I\right\}$ is a family of superadditive lower semicontinuous and $\mathbb{Q}_{+}^{-}$ homogeneous set-valued functions $F_{i}: K \rightarrow n(Y)$. If $K$ is of the second category in $K$ and $\bigcup_{i \in I} F_{i}(x) \in b(Y)$ for $x \in K$, then there exists a positive constant $M$ such that

$$
\left\|F_{i}(x)\right\|:=\sup \left\{\|y\|: y \in F_{i}(x)\right\} \leq M\|x\|
$$

for every $i \in I$ and $x \in K$.

Corollary 1. If $X, Y$ and $K$ are as in Lemma 3 , then the functional

$$
F \mapsto\|F\|:=\sup \{\|F(x)\| /\|x\|: x \in K, x \neq 0\}
$$


is finite for every $\mathbb{Q}_{+}$-homogeneous superadditive lower semicontinuous setvalued function $F: K \rightarrow b(Y)$.

Lemma 4 (Lemma 5 in [9]). Let $X$ and $Y$ be two normed spaces and let $d$ be the Hausdorff distance derived from the norm in $Y$. Suppose that $K$ is a convex cone in $X$ with nonempty interior. Then there exists a positive constant $M_{0}$ such that for every linear continuous set-valued function $F$ : $K \rightarrow c(Y)$ the inequality

$$
d(F(x), F(y)) \leq M_{0}\|F\|\|x-y\|
$$

holds for every $x, y \in K$.

Lemma 5 (Theorem 2 in [5]). Let $\left(X, \varrho_{X}\right)$ and $\left(Y, \varrho_{Y}\right)$ be two metric spaces and let $d_{X}$ and $d_{Y}$ be the corresponding Hausdorff metrics. If $F$ : $X \rightarrow n(Y)$ is a set-valued function and $M$ is a positive constant such that

$$
d_{Y}(F(x), F(y)) \leq M \varrho_{X}(x, y)
$$

for any $x, y \in X$, then

$$
d_{Y}(F(A), F(B)) \leq M d_{X}(A, B)
$$

for any nonempty subsets $A, B$ of $X$.

Lemma 6 (Lemma 4 in [8]). Let $D$ be a nonempty set and $Y$ be a normed space. If $F_{0}, F_{n}: D \rightarrow c(Y)$ are set-valued functions and the sequence $\left(F_{n}\right)$ uniformly converges to $F_{0}$ on $D$, then

$$
\lim _{n \rightarrow \infty} F_{n}(D)=F_{0}(D) .
$$

Lemma 7. Let $X$ be a Banach space, $Y$ a normed space, and $K$ a closed convex cone in $X$ with nonempty interior. Suppose that $F_{0}, F_{n}: K \rightarrow c(Y)$ are continuous linear set-valued functions. If

$$
F_{0}(y)=\lim _{n \rightarrow \infty} F_{n}(y)
$$

for $y \in K$, then the sequence $\left(F_{n}\right)$ uniformly converges to $F_{0}$ on every $D \in c(K)$.

Proof. By (1) the set $\bigcup\left\{F_{n}(y): n=0,1,2, \ldots\right\}$ is bounded for every $y \in K$ and by Lemma 3 there exists a positive constant $M$ such that

$$
\left\|F_{n}\right\| \leq M
$$

for $n=0,1,2, \ldots$ According to Lemma 4 there exists a positive constant $M_{0}$ such that

$$
d\left(F_{n}(x), F_{n}(y) \leq M_{0}\left\|F_{n}\right\|\|x-y\| \leq M_{0} M\|x-y\|\right.
$$

for $x, y \in K$ and $n=0,1,2, \ldots$ This implies that

$$
\begin{aligned}
& \left|d\left(F_{n}(x), F_{0}(x)\right)-d\left(F_{n}(y), F_{0}(y)\right)\right| \\
& \leq d\left(F_{n}(x), F_{n}(y)\right)+d\left(F_{0}(x), F_{0}(y)\right) \leq 2 M M_{0}\|x-y\| .
\end{aligned}
$$


Consequently, the family $\left\{d\left(\left(F_{n}(\cdot), F_{0}(\cdot)\right): n=0,1,2, \ldots\right\}\right.$ is equicontinuous in $K$ and by (1), $F_{0}$ is the uniform limit of $\left(F_{n}\right)$ on every compact subset $D$ of $K$ (see Theorem 3.2.4 in [4]).

3. Now, we can prove our main results.

TheOREM 1. Let $X$ be a Banach space and let $K$ be a closed convex cone in $X$ with nonempty interior. Suppose that $\left\{F^{t}: t \geq 0\right\}$ is a differentiable iteration semigroup of linear continuous set-valued functions $F^{t}: K \rightarrow c c(K)$ with $F^{0}(x)=\{x\}$. Then the set-valued function $(t, x) \mapsto F^{t}(x)$ is continuous and

$$
D_{t} F^{t}(x)=F^{t}(G(x))
$$

for $x \in K, t \geq 0$, where $D_{t}$ denotes the Hukuhara derivative of $F^{t}(x)$ with respect to $t$ and

$$
G(x):=\lim _{s \rightarrow 0+} \frac{F^{s}(x)-x}{s}
$$

for $x \in K$.

Proof. It is obvious that the differences $F^{s}(x)-x$ exist for $s>0$ and $x \in K$, and hence, according to Lemma 2 , so do the differences

$$
F^{t+s}(x)-F^{t}(x)=F^{t}\left[F^{s}(x)\right]-F^{t}(x)=F^{t}\left(F^{s}(x)-x\right)
$$

and

$$
F^{t}(x)-F^{t-s}(x)=F^{t-s}\left[F^{s}(x)\right]-F^{t-s}(x)=F^{t-s}\left(F^{s}(x)-x\right)
$$

whenever $t>0, s \in(0, t)$ and $x \in K$.

By Lemmas 4 and 5 ,

$$
\begin{aligned}
d\left(\frac{F^{t+s}(x)-F^{t}(x)}{s}, F^{t}(G(x))\right) & =d\left(F^{t}\left(\frac{F^{s}(x)-x}{s}\right), F^{t}(G(x))\right) \\
& \leq M_{0}\left\|F^{t}\right\| d\left(\frac{F^{s}(x)-x}{s}, G(x)\right)
\end{aligned}
$$

for $x \in K, t>0, s \in(0, t)$, so (2) implies

$$
\lim _{s \rightarrow 0+} \frac{F^{t+s}(x)-F^{t}(x)}{s}=F^{t}(G(x))
$$

for $t>0$ and $x \in K$.

Similarly, for $t>0, s \in(0, t)$ and $x \in K$ we have

$$
\begin{aligned}
d\left(\frac{F^{t}(x)-F^{t-s}(x)}{s},\right. & \left.F^{t}(G(x))\right) \\
& =d\left(F^{t-s}\left(\frac{F^{s}(x)-x}{s}\right), F^{t-s}\left(F^{s}(G(x))\right)\right) \\
\leq & M_{0}\left\|F^{t-s}\right\| d\left(\frac{F^{s}(x)-x}{s}, F^{s}(G(x))\right) .
\end{aligned}
$$


By Lemma 1 the function $s \mapsto F^{s}(y)$ is continuous for every $y \in K$. Therefore the image $\bigcup_{0 \leq s \leq t} F^{s}(y)$ of the interval $[0, t]$ under this set-valued function is compact (see [1, p. 110]), whence it is bounded. According to Lemma 3 there exists a positive constant $M$ such that

$$
\left\|F^{t-s}(y)\right\| \leq M\|y\|
$$

for $0 \leq s \leq t$ and $y \in K$. Consequently,

$$
\left\|F^{t-s}\right\| \leq M
$$

for $s \in[0, t]$. This inequality and (3) imply that

$$
\begin{aligned}
& d\left(\frac{F^{t}(x)-F^{t-s}(x)}{s}, F^{t}(G(x))\right) \\
& \leq M_{0} M d\left(\frac{F^{s}(x)-x}{s}, G(x)\right)+M_{0} M d\left(G(x), F^{s}(G(x))\right)
\end{aligned}
$$

for $t>0, s \in(0, t)$ and $x \in K$. By Lemmas 7 and 6 we have

$$
\lim _{s \rightarrow 0+} F^{s}(G(x))=G(x)
$$

and by (2) and (4),

$$
D_{t} F^{t}(x)=\lim _{s \rightarrow 0+} \frac{F^{t}(x)-F^{t-s}(x)}{s}=F^{t}(G(x)) .
$$

It remains to prove that the multifunction $(t, x) \mapsto F^{t}(x)$ is continuous. Fix $t \geq 0, x \in K$ and $y \in G(x)$. By Lemmas $3-5$ there are two positive constants $M_{0}$ and $M$ such that

$$
\begin{aligned}
d\left(F^{t+s}(z), F^{t}(y)\right) & \leq d\left(F^{t+s}(z), F^{t+s}(y)\right)+d\left(F^{t+s}(y), F^{t}(y)\right) \\
& \leq M_{0}\left\|F^{t}\right\|\left(M\|z-y\|+d\left(F^{s}(y),\{y\}\right)\right)
\end{aligned}
$$

for every $s \in(0,1)$ and $z \in G(x)$. Therefore

$$
\limsup _{(s, z) \rightarrow(0+, y)} d\left(F^{t+s}(z), F^{t}(y)\right)=0 .
$$

Similarly, fix $t>0, x \in K$ and $y \in G(x)$. There exist two positive constants $M_{0}$ and $M_{1}$ for which

$$
\begin{aligned}
d\left(F^{t-s}(z), F^{t}(y)\right) & \leq d\left(F^{t-s}(z), F^{t-s}(y)\right)+d\left(F^{t-s}(y), F^{t}(y)\right) \\
& \leq M_{0} M_{1}\left(\|z-y\|+d\left(F^{s}(y),\{y\}\right)\right),
\end{aligned}
$$

for every $s \in(0, t)$ and $z \in G(x)$. By (5) and (6) the set-valued function $(t, y) \mapsto F^{t}(y)$ is continuous.

Definition 1. Let $K$ be a convex cone in a Banach space $X$ and let $G, \Psi: K \rightarrow c c(K)$ be two continuous linear maps. A map $\Phi:[0, \infty) \times K \rightarrow$ $c c(K)$ is said to be a solution of the problem

$$
D_{t} \Phi(t, x)=\Phi(t, G(x)):=\bigcup\{\Phi(t, y): y \in G(x)\},
$$




$$
\Phi(0, x)=\Psi(x)
$$

if $\Phi$ is continuous in $[0, \infty) \times K$ and differentiable with respect to $t$, and satisfies $(7)$ and (8) everywhere in $[0, \infty) \times K$ and $K$, respectively.

Lemma 8 (Theorem 2 in [11]). Let $K$ be a closed convex cone with nonempty interior in a Banach space and let $G, \Psi: K \rightarrow c c(K)$ be two continuous linear maps. Then there exists exactly one solution of problem (7)-(8). This solution is linear with respect to the second variable.

Theorem 2. Let $X$ be a Banach space, let $K$ be a closed convex cone in $X$ with nonempty interior, and let $G: K \rightarrow c c(K)$ be a continuous linear set-valued function. Suppose that $\Phi:[0, \infty) \times K \rightarrow c c(K)$ is a solution of problem (7)-(8) with $\Psi(x)=\{x\}$ for $x \in K$, such that every set-valued function $x \mapsto \Phi(t, x), t \geq 0$, is linear. Then the family $\left\{F^{t}: t \geq 0\right\}$, where $F^{t}(x):=\Phi(t, x)$ for $(t, x) \in[0, \infty) \times K$, is a differentiable iteration semigroup.

Proof. Fix $t \geq 0$ and define

$$
\alpha(s, x):=\Phi(s+t, x), \quad \beta(s, x):=\Phi(t, \Phi(s, x))
$$

for $x \in K, s \geq 0$. We see that

$$
\begin{aligned}
& \alpha(0, x)=\Phi(t, x), \\
& \beta(0, x)=\Phi(t, \Phi(0, x))=\Phi(t, x)
\end{aligned}
$$

for $x \in K$. Now, we have

$$
\begin{array}{r}
\lim _{u \rightarrow s+} \frac{\alpha(u, x)-\alpha(s, x)}{u-s}=\lim _{u \rightarrow s+} \frac{\Phi(u+t, x)-\Phi(s+t, x)}{u-s}=\Phi(s+t, G(x)) \\
=\bigcup\{\Phi(s+t, y): y \in G(x)\}=\bigcup\{\alpha(s, y): y \in G(x)\}=\alpha(s, G(x))
\end{array}
$$

for $x \in K, s \geq 0$, and

$$
\begin{aligned}
\lim _{u \rightarrow s_{-}} \frac{\alpha(s, x)-\alpha(u, x)}{s-u} & =\lim _{u \rightarrow s-} \frac{\Phi(s+t, x)-\Phi(u+t, x)}{s-u} \\
& =\Phi(s+t, G(x))=\alpha(s, G(x))
\end{aligned}
$$

for $x \in K, s>0$. Thus

$$
D_{s} \alpha(s, x)=\alpha(s, G(x))
$$

for $x \in K, s \geq 0$. Further, by Lemma 2 we have

$$
\begin{aligned}
\frac{\beta(u, x)-\beta(s, x)}{u-s} & =\frac{\Phi(t, \Phi(u, x))-\Phi(t, \Phi(s, x))}{u-s} \\
& =\Phi\left(t, \frac{\Phi(u, x)-\Phi(s, x)}{u-s}\right)
\end{aligned}
$$


for $u>s \geq 0, x \in K$, and according to Lemma 4 ,

$$
\begin{aligned}
d\left(\frac{\beta(u, x)-\beta(s, x)}{u-s},\right. & \left.\Phi\left(t, D_{s} \Phi(s, x)\right)\right) \\
& =d\left(\Phi\left(t, \frac{\Phi(u, x)-\Phi(s, x)}{u-s}\right), \Phi\left(t, D_{s} \Phi(s, x)\right)\right) \\
& \leq M_{0}\|\Phi(t, \cdot)\| d\left(\frac{\Phi(u, x)-\Phi(s, x)}{u-s}, D_{s} \Phi(s, x)\right) .
\end{aligned}
$$

Thus

$$
\begin{gathered}
\lim _{u \rightarrow s+} \frac{\beta(u, x)-\beta(s, x)}{u-s}=\Phi\left(t, D_{s} \Phi(s, x)\right)=\Phi(t, \Phi(s, G(x))) \\
=\Phi(t, \bigcup\{\Phi(s, y): y \in G(x)\})=\bigcup\{\Phi(t, \Phi(s, y)): y \in G(x)\} \\
=\bigcup\{\beta(s, y): y \in G(x)\}=\beta(s, G(x)) .
\end{gathered}
$$

Similarly for $s>u \geq 0, x \in K$, we have

$$
\begin{aligned}
\frac{\beta(s, x)-\beta(u, x)}{s-u} & =\frac{\Phi(t, \Phi(s, x))-\Phi(t, \Phi(u, x))}{s-u} \\
& =\Phi\left(t, \frac{\Phi(s, x)-\Phi(u, x)}{s-u}\right) .
\end{aligned}
$$

and

$$
\begin{aligned}
d\left(\frac{\beta(s, x)-\beta(u, x)}{s-u},\right. & \left.\Phi\left(t, D_{s} \Phi(s, x)\right)\right) \\
& =d\left(\Phi\left(t, \frac{\Phi(s, x)-\Phi(u, x)}{s-u}\right), \Phi\left(t, D_{s} \Phi(s, x)\right)\right) \\
& \leq M_{0}\|\Phi(t, \cdot)\| d\left(\frac{\Phi(s, x)-\Phi(u, x)}{s-u}, D_{s} \Phi(s, x)\right) .
\end{aligned}
$$

Thus

$$
\begin{gathered}
\lim _{u \rightarrow s-} \frac{\beta(s, x)-\beta(u, x)}{s-u}=\Phi\left(t, D_{s} \Phi(s, x)\right)=\Phi(t, \Phi(s, G(x))) \\
=\Phi(t, \bigcup\{\Phi(s, y): y \in G(x)\})=\bigcup\{\Phi(t, \Phi(s, y)): y \in G(x)\} \\
=\bigcup\{\beta(s, y): y \in G(x)\}=\beta(s, G(x)) .
\end{gathered}
$$

Therefore

$$
D_{s} \beta(s, x)=\beta(s, G(x)) .
$$

Equalities (9)-(12) mean that $\alpha$ and $\beta$ are solutions of problem (7)-(8) with $\Psi(x)=\Phi(t, x)$. By Lemma 8 a solution of $(7)-(8)$ is unique. Consequently, $\beta=\alpha$, which means that

$$
F^{t}\left(F^{s}(x)\right)=\Phi(t, \Phi(s, x))=\Phi(t+s, x)=F^{t+s}(x) .
$$

This completes the proof. 
4. Now, we give some applications.

Corollary 2. Let $K$ be a closed convex cone with nonempty interior in a Banach space and let $\left\{F^{t}: t \geq 0\right\}$ be a concave iteration semigroup of continuous linear set-valued functions $F^{t}: K \rightarrow c c(K)$ with $F^{0}(x)=\{x\}$ for $x \in K$. Then the set-valued function $\Phi:[0, \infty) \times K \rightarrow c c(K), \Phi(t, x)=F^{t}(x)$, is a solution of problem (7)-(8) with $\Psi(x)=\{x\}$ for $x \in K$, where $G$ is given by (2), and the set-valued functions $t \mapsto \Phi(t, G(x)), x \in K$, are increasing.

Proof. By the Theorem in [10] the iteration semigroup $\left\{F^{t}: t \geq 0\right\}$ is differentiable and the set-valued function $\Phi(t, x):=F^{t}(x)$ is a solution of problem (7)-(8) with $\Psi(x)=\{x\}$, where $G$ is given by (2). Since the set-valued functions $t \mapsto \Phi(t, x), x \in K$, are concave, Theorem 3.1 in [6] implies that the set-valued functions $t \mapsto D_{t} \Phi(t, x)$ are increasing. Thus the set-valued functions $t \mapsto F^{t}(G(x)), x \in K$, are also increasing.

COROllary 3. Let $K$ be a closed convex cone with nonempty interior in a Banach space and let $G: K \rightarrow c c(K)$ be a continuous linear set-valued function. If $\Phi:[0, \infty) \times K \rightarrow c c(K)$ is a solution of problem (7)-(8) with $\Psi(x)=\{x\}$ for $x \in K$, such that the set-valued functions $x \mapsto \Phi(t, x), t \geq 0$, are linear and the functions $t \mapsto \Phi(t, G(x)), x \in K$, are increasing, then the family $\left\{F^{t}: t \geq 0\right\}$, where $F^{t}(x):=\Phi(t, x)$, is a concave iteration semigroup of continuous linear set-valued functions.

Proof. By Theorem 2 the family $\left\{F^{t}: t \geq 0\right\}$ is a differentiable iteration semigroup. According to the Proposition in [11],

$$
F^{t}(x)=\Phi(t, x)=x+\int_{0}^{t} \Phi(s, G(x)) d s
$$

for $x \in K, t \geq 0$, where $\int_{0}^{t}$ denotes a Riemann-type integral. Since the setvalued functions $s \mapsto \Phi(s, G(x))$ are increasing, Corollary 4.4 in [6] implies that the set-valued functions $t \mapsto F^{t}(x), x \in K$, are concave. That means that the iteration semigroup $\left\{F^{t}: t \geq 0\right\}$ is concave.

Corollary 4. Let $K$ be a closed convex cone with nonempty interior in a Banach space. Suppose that $\left\{F^{t}: t \geq 0\right\}$ is a differentiable iteration semigroup of continuous linear set-valued functions $F^{t}: K \rightarrow c c(K)$ with $F^{0}(x)=\{x\}$ for $x \in K$. Then this semigroup is increasing if and only if $0 \in G(x)$ for $x \in K$.

Proof. Suppose that $\left\{F^{t}: t \geq 0\right\}$ is increasing. Then $x \in F^{t}(x)$ for $x \in K$ and

$$
0 \in G(x)=\lim _{t \rightarrow 0+} \frac{F^{t}(x)-x}{t}
$$

for $x \in K, t \geq 0$. 
To prove the converse, note that by Theorem 1 the set-valued function $\Phi(t, x):=F^{t}(x)$ is a solution of problem (7)-(8) with $\Psi(x)=\{x\}$ and by the Proposition in [11],

$$
F^{t}(x)=x+\int_{0}^{t} F^{s}(G(x)) d s .
$$

If (13) holds then by (14), $x \in F^{t}(x)$ for $x \in K, t \geq 0$, so this semigroup is increasing.

\section{References}

[1] C. Berge, Topological Spaces, Oliver and Boyd, Edinburgh and London, 1963.

[2] H. Frankowska, Local controllability and infinitesimal generators of semigroups of set-valued maps, SIAM J. Control Optim. 25 (1987), 412-432.

[3] M. Hukuhara, Intégration des applications mesurables dont la valeur est un compact convexe, Funkcial. Ekvac. 10 (1967), 205-223.

[4] S. Łojasiewicz, An Introduction to the Theory of Real Functions, Wiley, 1988.

[5] S. B. Nadler, Jr., Multi-valued contraction mappings, Pacific J. Math. 30 (1969), 475-488.

[6] M. Piszczek, Integral representations of convex and concave set-valued functions, Demonstratio Math. 35 (2002), 727-742.

[7] H. Rådström, An embedding theorem for spaces of convex sets, Proc. Amer. Math. Soc. 3 (1952), 165-169.

[8] A. Smajdor, Increasing iteration semigroups of Jensen set-valued functions, Aequationes Math. 56 (1998), 131-142.

[9] —, On regular multivalued cosine families, Ann. Math. Sil. 13 (1999), 271-280.

[10] - Hukuhara's derivative and concave iteration semigroups of linear set-valued functions, J. Appl. Anal. 8 (2002), 297-305.

[11] -, On a multivalued differential problem, Internat. J. Bifur. Chaos Appl. Sci. Engrg. 13 (2003), 1877-1882.

[12] W. Smajdor, Superadditive set-valued functions and Banach-Steinhaus theorem, Rad. Mat. 3 (1987), 203-214.

[13] P. R. Wolenski, A uniqueness theorem for differential inclusions, J. Differential Equations 84 (1990), 165-182.

Department of Mathematics

Pedagogical University

Podchorążych 2

30-084 Kraków, Poland

E-mail: asmajdo@wsp.krakow.pl

Reçu par la Rédaction le 21.3.2002

Révisé le 24.11.2003 